\title{
Changes in Peripapillar Retinal Nerve Fiber Layer Analized by Td-Oct in Patients with Diabetic Retinopathy That Receive Panretinal Photocoagulation
}

\author{
Manuel Eliezer Manzanero Hernandez \\ Department of Ophthalmology, University of Guadalajara, Regional Hospital "Valentin Gomez Farias", Jalisco, Mexico
}

\section{Email address:}

eliezermh@msn.com

\section{To cite this article:}

Manuel Eliezer Manzanero Hernandez. Changes in Peripapillar Retinal Nerve Fiber Layer Analized by Td-Oct in Patients with Diabetic Retinopathy That Receive Panretinal Photocoagulation. International Journal of Ophthalmology \& Visual Science. Vol. 3, No. 1, 2018 , pp. 1-6. doi: $10.11648 /$ j.ijovs.20180301.11

Received: October 14, 2017; Accepted: October 26, 2017; Published: December 5, 2017

\begin{abstract}
In this study, changes in Retinal Nerve Fiber Layer (RNFL) within peripapillar zone in patients with diabetic retinopathy that accomplish criteria to receive panretinal photocoagulation are analyzed by TD-OCT (Time Domain-Optic Coherence Tomography) unit. This analysis was transversal retrospective and observational and was done in 46 eyes from 33 patients. According to laser application technique, time since application and day of the study, 4 groups of patients were set: the first with patients with less than 30 days since last laser application and day of study. The second with patients with 180 days since laser photocoagulation; the last two groups received panretinal photocoagulation plus macular selective laser with a TD-OCT performed 30 days (for the third group) and 180 days (the fourth group) after administration of treatment. The clinically significant finding was in comparing the group that received panretinal photocoagulation with TD-OCT after 30 days, and the group that received panretinal photocoagulation and TD-OCT after 180 days. Average thickness was less in the second group, with a statistical significant finding $\mathrm{p}=0.012$ in inferior quadrant. Administer laser photocoagulation makes changes in the structure and function of the retina, and according to references many different results may be possible taking in count the equipment used to apply treatment and to take measurements. The patient must be informed about secondary and adverse effects after the treatment
\end{abstract}

Keywords: Diabetic Retinopathy, Panretinal Photocoagulation, Macular Laser, Time-Domain Optic Coherence Tomography, Retnal Nerve Fiber Layer

\section{Introduction}

Diabetic Retinopathy is one of first causes of blindness or severe visual impairment, in Mexico has been reported up to $8 \%$ of blindness cases because of diabetic retinopathy [1]. In other countries as England, data are $14.4 \%$ to $17.7 \%$ in causes of blindness [2]. In both cases CIE-10 classification considers severe visual impairment that one with far visual acuity best or equal to $20 / 400$, but worse than $20 / 200$, or blindness is diagnosed when visual field is restricted only to 10 grades from central point of fixation in anyone of eyes or worst than 20/400 [3].

Administration of laser in diabetic retinopathy had an important evidence in the Early Treatment Diabetic Retinopathy Study (ETDRS), published in 1976, and with subsequent reports through years, with evidence that extensive laser application in retina and focal zones with neovascularization can reduce at $50 \%$ the risk of visual lost, at least in a following of 2 years [4]. After random studies, the ETDRS, through several subsequent reports from 1985, demonstrates that photocoagulation in patients with clinically significant diabetic macular edema reduces the risk of visual lost [5].

Indications for panretinal photocoagulation are: patients with proliferative diabetic retinopathy with or without high risk characteristics (optic disk neovascularization greater than a quarter of optic disk, or neovascularization not in optic disk but greater in size than a half disk diameter or with vitreous or preretinal hemorrhage) may be that the patient has or not central macular edema, or in patients with circumstances that difficult subsequent medical checkups such as severe diabetic retinopathy, a long distance home-laser unit, fast evolution 
disease, bad treatment accomplishment, or patients with mobilization problems [6].

In another way, the indication to administer macular selective laser is the presence of clinically significant macular edema, this particular pathology has specific characteristics: a) retinal thickening within 500 microns of the center of the fovea, b) hard exudates within 500 microns of the center of the fovea, with retinal thickening, c) one or more optic disc diameters of retinal thickening, part of which is within one disc diameter of the macula center. Just one of these criteria is enough to diagnostic. Additionally, there should be no vitreomacular traction [6].

\section{Material and Methods}

\subsection{Objective}

Describe the changes in retinal nerve thickness after administer panretinal photocoagulation in different groups of patient, each group with a different time lapse between laser administration and OCT study, the retinal nerve fiber layer thickness is compared with the data base of normal thickness that the OCT units has pre-installed [7].

\subsection{Population}

This study was under informed consent, developed in the ophthamology department of Regional Hospital Valentin Gomez Farias, Jalisco, Mexico. With approval of the Etic and Investigation Committee of the hospital and in base of Helsinsky declaration.

\subsubsection{Inclusion Criteria}

The inclusion criteria were patients with proliferative diabetic retinopathy with or without high risk criteria, from April 4, to July 29 of 2016, in these patient photocoagulation was administered in the wider possible area.

\subsubsection{Exclusion Criteria}

Exclusion criteria were patients with glaucoma, high density cataract, hemovitreous, or a bad quality signal in OCT unit (less than 6).

\subsection{Techniques and Equipment}

\subsubsection{Laser Unit}

Photocoagulation was done with Argon Carl Zeiss Visulass 532s LASER, for such procedure, an Ocular 165 lens was used, with previous topical anesthesia, and hypromellose $2 \%$ was set in the lens previous contact with the surface of the eye. In total, 3 or less sessions were required to complete treatment, with a difference of 1 week between each one, patients who receive macular selective laser had clinically significant macular edema without vitreomacular traction.

\subsubsection{TD-OCT Unit}

Measurements in selected patients were made with a Humphrey-Zeiss TD-OCT, Stratus model. All of them received tropicamide plus epinephrine to get mydriasis, with 1 drop in the selected eye.

\subsection{Statistical Analysis}

A transversal, retrospective and observational study was done to analyze peripapillar RNFL in patients with diabetic retinopathy who received panretinal photocoagulation. The number of patients was in base of convenience because of the patients had irregularities in subsequent medical checkups.

The computer program used for analysis of patients was SPSS v.20 for MAC. Chi-squared test and contingency table were used for categorical variables, with confidence intervals within $95 \%$, taking a statistically significant value a $\mathrm{p}<0.05$. Non-parametric Kolmogorov-Smirnov test for normality was calculated in case of numerical variables. Also, were compared statistical mean with ANOVA and t-test according to situation.

\section{Results}

\subsection{Characteristics and Division by Group}

The selection was in a base of 40 patients whose were gotten 79 eyes to take TD-OCT, 1 patient had 1 eye secondary to ocular trauma. Applying exclusion criteria, final available patients were 33, with 46 eyes in total; In these 33 patients, 24 men, 9 women, with average age of 60.03 years old for women and 56.5 for men, a total average of 58.9 years old for both genders. These 46 eyes were segmented in 4 groups according to laser administration technique and elapsed time since last application. To simplify descriptions, abbreviations are used for each group:

Total of laser shots received were registered in each one, and average thickness of retinal nerve fiber layer in each quadrant displayed in TD-OCT unit: superior, nasal, inferior, temporal.

1. -The first group (PPC30) received panretinal photocoagulation within 30 days since the last session, getting 3 eyes, from 20 patients. Average laser shots were 1931, with 13 right eyes, 17 left eyes.

2. - The second group (PPC180) were patients that received panretinal photocoagulation 180 days previous OCT study, included 10 eyes from 8 patients.

3. -Third group (MSL30) included patients that received photocoagulation in retina plus macular selective laser, 180 days before OCT study, were included 3 patients, 3 right eyes and 1 left eye.

4. -The fourth and last group (MSL180) were patients with photocoagulation in retina plus macular selective laser administered 180 days before OCT study. Included 2 eyes, from 1 patient

\subsection{Analysis and Results by Group}

In each patient was compared mean value of each quadrant with the thickness of RNFL in base of the TD-OCT manual from the unit software. First were compared mean thickness of all the groups with non-parametric Kolmogorov-Smirnov test for normality. The mean values were compared with ANOVA test. There were not statistically significant $\mathrm{P}$ values (table 1). 
Table 1. Nerve fiber layer thickness average in patient groups, in each quadrant, with average shots of laser.

\begin{tabular}{llllll}
\hline Variables & PPC30(SD) & PPC180(SD) & MSL30(SD) & MSL180(SD) \\
\hline $\begin{array}{l}\text { Average laser shots } \\
\text { Fiber thicknes (micras) }\end{array}$ & $1931.10( \pm 1019.05)$ & $2426.3( \pm 613.9)$ & $1595.7( \pm 1118.52)$ & $495( \pm 388.9)$ \\
Superior & $116.97( \pm 44.12) \mu$ & $88.10( \pm 14.7) \mu$ & $85.7( \pm 11.7) \mu$ & & $104.5( \pm 4.9) \mu$ \\
Nasal & $84.2( \pm 35.21) \mu$ & $63.8( \pm 21.8) \mu$ & $75( \pm 13.39) \mu$ & $67.5( \pm 13.43) \mu$ & 0.130 \\
Inferior & $120.23( \pm 29.96) \mu$ & $93.5( \pm 18.65) \mu$ & $111.5( \pm 27.16) \mu$ & $129( \pm 8.45) \mu$ & 0.332 \\
Temporal & $91.9( \pm 45.4) \mu$ & $71.6( \pm 23.01) \mu$ & $72.25( \pm 25.39) \mu$ & $63( \pm 21.21) \mu$ & 0.65 \\
\hline
\end{tabular}

Kolmogorov-smirnov test was used for normality. To get "p" value, the mean was compared with ANOVA

SD: Standard deviation

$\mu$ : Microns

Comparing each quadrant of each patient with the TD-OCT data base, they were grouped in low, normal, or high, this process was made in all the 4 groups. In the final result there was not a significant $p$ (table 2). Contingence tables were used in group comparison, using chi-square test.

Table 2. Eyes are assigned in 3 groups: low, normal, or high, considering retina nerve fiber layer thickness in each quadrant, and laser administration characteristics.

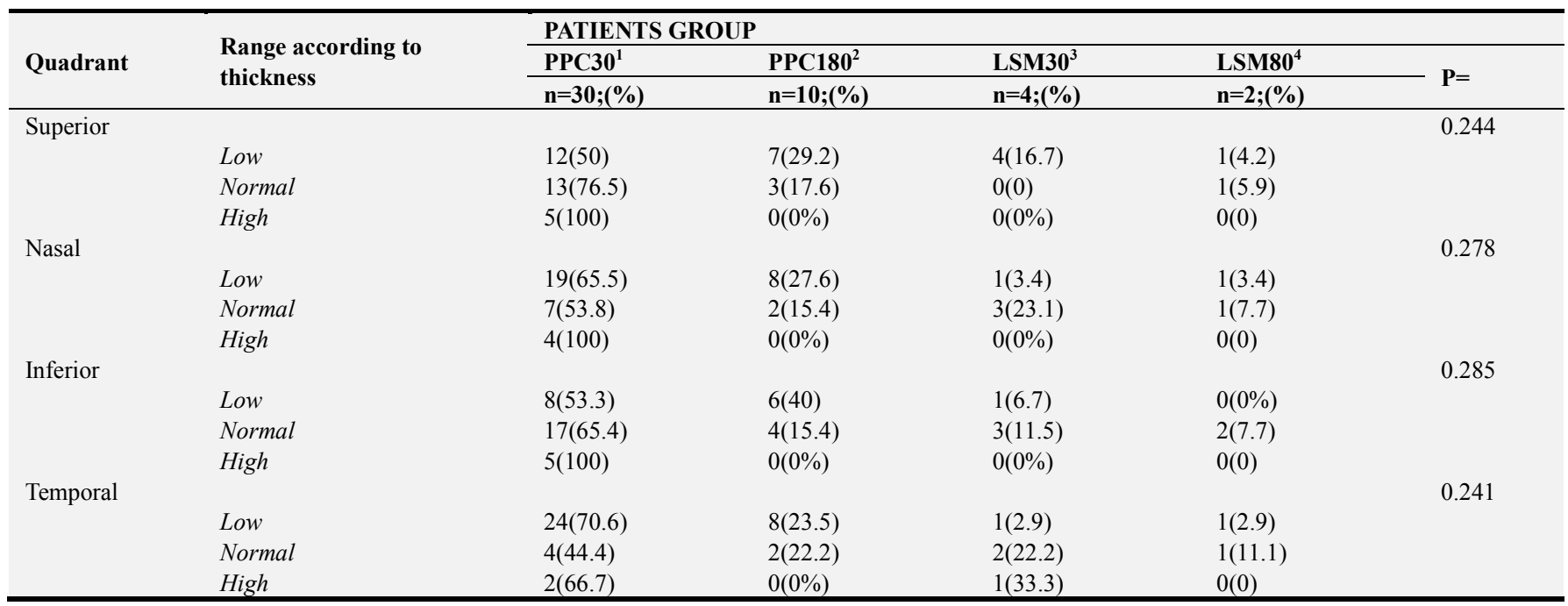

Contingency tables were used, comparing groups with chi-square test, with confident interval of $95 \%$, and a significative result a $\mathrm{p}<0.05$.

1: Group with an OCT 30 days after panretinal photocoagulation

2: Group with an OCT 180 days after panretinal photocoagulation

3: Group with an OCT 30 days after panretinal photocoagulation plus macular selective laser

4: Group with an OCT 180 days after panretinal photocoagulation plus macular selective laser

$\mathrm{n}$ : number of patients

A non-parametric Kolmogorov-Smirnov test for normality was made to compare the mean thickness of each quadrant in PPC30 and PPC180 groups. In this case there was a statistical significant result of $p=0.012$ in inferior quadrant; In PFC180 group was found a low mean in the nasal quadrant (Table 3) situation that will be analyzed in the discussion.

Table 3. Comparison of nerve fiber layer thickness between PFC30 and PFC180 group, both without macular selective laser.

\begin{tabular}{|c|c|c|c|}
\hline & $\mathrm{PPC30}^{1}$ & $\mathrm{PPC}^{180^{2}}$ & \\
\hline & $\left(\mathrm{SD}^{3}\right)$ & (SD) & $\mathbf{P}$ \\
\hline Average laser shots & $1931.10( \pm 1019.05)$ & $2426.3( \pm 613.9)$ & \\
\hline \multicolumn{4}{|l|}{ Quadrant } \\
\hline Superior & $116.97( \pm 44.12) \mu$ & $88.10( \pm 14.7) \mu$ & 0.051 \\
\hline Nasal & $84.2( \pm 35.21) \mu$ & $63.8( \pm 21.8) \mu$ & 0.094 \\
\hline Inferior & $120.23( \pm 29.96) \mu$ & $93.5( \pm 18.65) \mu$ & 0.012 \\
\hline Temporal & $91.9( \pm 45.4) \mu$ & $71.6( \pm 23.01) \mu$ & 0.185 \\
\hline
\end{tabular}

In case of numerical variables, Kolmogorov-Smirnov test for normality was used. The mean was compared with t-test.

1: Group with an OCT 30 days after panretinal photocoagulation

2: Group with an OCT 180 days after panretinal photocoagulation

3: standard deviation

$\mu$ : Microns 
A comparison between thickness of MSL30 and MSL180 group was realized. Using non parametric Kolmogorov-Smirnov test for normality, and t-test for mean values, without results statistically significant (Table 4).

Table 4. Comparison of retinal nerve fiber thickness of MSL30 y MSL180 group.

\begin{tabular}{llll}
\hline & LSM30 $^{1}$ (SD $^{3}$ ) & LSM180 $^{2}$ (SD) & P= \\
\hline Averages laser shots & $1595.7( \pm 118.52)$ & $495( \pm 388.9)$ & \\
Quadrant & & & \\
Superior & $85.7( \pm 11.7) \mu$ & $104.5( \pm 4.9) \mu$ & 0.107 \\
Nasal & $75( \pm 13.39) \mu$ & $67.5( \pm 13.43) \mu$ & 0.553 \\
Inferior & $111.5( \pm 27.16) \mu$ & $129( \pm 8.45) \mu$ & 0.445 \\
Temporal & $72.25( \pm 25.39) \mu$ & $63( \pm 21.21) \mu$ & 0.684 \\
\hline
\end{tabular}

In case of numerical variables, Kolmogorov-Smirnov test for normality was used. The mean was compared with t-test.

1: Group with an OCT 30 days after panretinal photocoagulation plus macular selective laser

2: Group with an OCT 180 days after panretinal photocoagulation plus macular selective laser.

3: Standard deviation

$\mu$ : microns

\section{Discussion}

Secondary effects after photocoagulation in retina have been described, including defects in campimetries from 15 to 30 grades within central vision field, with most of affection if the laser spots are near of peripapillar zone [8]; a thicker central macular area may originates edema, and vitreous hemorrhage [9]; peripheral and night vision loss are significant effects, as well as a decrement in 1 to 4 lines of central vision in $10 \%$ of patients, this secondary effect is seen more frequently in patients with high risk criteria, and not so common in persons with epiretinal membrane, cataract, neovascular glaucoma, and macular degeneration [10].

Histological effects of laser are the result of retinal pigment epithelium and photoreceptors destruction and sometimes, even choriocapillaris damage. The resulting scare is formed by glia, with a few mitochondria and a consecutive low energy use, which lets oxygen a free flow to adjacent photoreceptors, the hydrostatic pressure decrements in capillary and venule secondary to capillary constriction, therefore edema decreases, because of best oxygenation and less capillary diameter [11].

Structurally, it has been corroborated with SD-OCT (Spectral Domain-Optic Coherence Tomography) focal necrosis areas that include inner nuclear layer and outer nuclear layer, an edema area is created a few hours after administered energy, with reabsorption in a couple of days. An atrophic scare will be formed with higher density in retinal pigment epithelium [12]. One month after a misaligned patron can be observed in any retina layer, that gradually will be homogeneous again, at least partially, but the scare will be thicker; is notable the direct relation between laser power and retinal damage in many studies by SD-OCT [13].

In this study, data analysis shows nerve fiber layer thickness changes in the group with panretinal phocoagulation within 30 days compared with the group that had more than 180 days at the OCT study time, with a lower thickness in this last group. Even if the mean thickness was lower in every quadrant, the significant difference was lesser in the inferior zone with a $\mathrm{p}=0.012$. In the listed references is found that 1 to 3 months post laser photocoagulation in patients with diabetic retinopathy the biggest change is a thicker retina [13], [14]. Although these characteristics were observable with Pascal Laser system, which has less effects in retina because of advantages with automatization in administered power and its distribution in an area; in this study Argon Laser was used.

Similarly, several studies demonstrate that peripapillar nerve fiber layer get thinner in 3 months [15], [16]. But this results were investigated in patients treated with PASCAL laser, also there is an article where Argon Laser was used (the same used in this article) and after a month a thinner layer is found [17], which correlates with this study.

In this study, 10 eyes with panretinal photocoagulation within 180 days since laser treatment had a tendency to be grouped in lower nerve fiber layer thickness; when these were compared with OCT data base, a total of 7 eyes had a lower thickness in superior quadrant, 8 eyes in nasal, 6 eyes in inferior, and 8 eyes in temporal, in spite of the statistically results were not significative, it is possible observe that total of eyes with lower thickness is greater than normal eyes, and there were not high thickness values. In reviewed articles, there was a significant statistically thinning in mean thickness of eyes with panretinal photocoagulation, but again, with Laser PASCAL unit. In the studies that used Argon Laser unit [17], [18], tendency to thinning of nerve fiber layers is confirmed.

Respect to comparison between panretinal photocoagulation with TD-OCT study at 30 days (PFC30) and panretinal photocoagulation plus macular selective laser with OCT study at 30 days (LSM30), the patients of the first group had tendency to stay with normal mean values, and the other group had tendency to mean thinner values, especially in superior quadrant. In the review of previous articles, it is told that if macular selective laser is administered, the most important changes are in inferior and superior quadrants [15], [19].

Comparing PFC30 group with PFC 180 group the mean of thickness was lower in this last group, all quadrants had a lower mean, but the significant difference was found in the inferior quadrant with a $p=0.012$. In reviewed references, 1 to 3 months after laser administration in patients with diabetic retinopathy the more relevant change is a thicker retina nerve fiber layer, but this result are found with PASCAL Laser unit, and as mentioned before, there are less secondary effects because of power and distribution of this automatized system; in the same way, may progress to thinner layer at long term, being more frequent in superior and inferior quadrant [15], [16], [17], [18]. There are many results in the following of the patients, either by the laser system, or by evolution time, even by specific patient conditions.

To analyze results of this study must be considered the Laser system unit and the results with previous investigations, because in some cases Pascal Laser unit is used, this system has the option to administer a time shot near 10 to 30 milliseconds, 
in a quantity of 1 to 56 in each pedal activation, and the Argon Laser system has longer times of administration, near 100 to 200 milliseconds, and only one shot at the time, in both cases is possible graduate the level of power in accord the spot size, this is under medical criteria [19]. Just a little variation has been seen in macular thickness in 6 months in patients that received laser with PASCAL system, because of less absorbed energy [20], nevertheless in revision articles final results suggest a tendency to be similar in retinal nerve fiber layer thickness in both laser system after 6 months of administration [21].

A limitation to compare this study with the ones in named references is that TD-OCT unit was used, while in the most articles the SD-OCT is used, and even some of those units are high definition. In the TD-OCT unit, the data obtained are fewer because of hardware limitations and a basic analysis logarithm [22].

Other articles point to the development of thinner nerve fiber layer in diabetic patients without photocoagulation compared with non diabetic patients, but photocoagulated patients have even thinner nerve fiber layer, that means that there is a tendency to thinner nerve fiber layer in diabetic patients [19].

\section{Conclusion}

References particularize that anatomical results are variable. Most cases the nerve fiber layer get thicker at 10 weeks, and thinner at 6 months after laser photocoagulation. Must be considered that some articles (just a few) explain that there is not difference between retinal thickness pre and post treatment, this evidences that results may vary. In this study, the results are alike references taking in count adverse anatomy effects in retina in the PFC30 and the PFC180 group. Also, must be considered geography condition and patient attitude in the geographical region to get subsequent revisions, because of their irregularity.

Results denote that there are changes in anatomy of the retina after laser treatment, particularly in short time group (PFC30), and must be considered that the equipment used in the following and treatment is not the best qualified, but it is important the correct interpretation and treatment decision no matter the resources. Obtained data show a perspective in the possibility to find information that can be interpreted for a best medical practice.

The secondary anatomical and functional changes described after energy administration in retina are a visual health problem, especially at long term like peripheral, night and central vision defects, plus natural history of diabetic retinopathy even under treatment, so, the patient must be informed about these adverse effects. The subsequent measurement of RNFL could be used as a valuable predictor in these problems.

\section{References}

[1] Sarah Polack, David Yorston, Antonio Lopez-Ramos, Sergio Lepe-Orta, Rogerio Martins Baia, Luciano Alveset al. Rapid Assessment of Avoidable Blindness and Diabetic Retinopathy in Chiapas, Mexico. Ophthalmology. 2012; 119:1033-40.
[2] Gerald Liew, Michell Michaelides, Catey Bounce. A comparison of the causes of blindness certifications in England and Wales in working age adults (16-64 years), 1999-2000 with 2009-2010. BMJ open 2014; 4(2):1-6.

[3] World Health Organization. ICD-10 International Statistical Classification of Diseases and Related Health Problems. 10th revisión, 2nd ed. Vol. 2, Blindness and low visión. Geneva Switzerland; WHO; 2010. ISBN: 9789241548342.

[4] Photocoagulation treatment of proliferative diabetic retinopathy. Clinical application of Diabetic Retinopathy Study (DRS) findings, DRS Report Number 8. The Diabetic Retinopathy Study Research Group. Ophthalmology. 1981; 88(7):583-600.

[5] Photocoagulation for diabetic macular edema. Early Treatment Diabetic Retinopathy Study report number 1. Early Treatment Diabetic Retinopathy Study research group. Archives of Ophthalmology. 1985; 103(12):1796-1806.

[6] Schlottmann P, Acosta C, Alezzandrini A. A, Befalluy J., Biccas L., Hidalgo Cano. Definición de estándares de buenas prácticas para el diagnóstico y tratamiento de la retinopatía diabética y el edema macular diabético en America Latina. The Pan-American Journal of Ophthalmology supplement. 2014; 13 suppl 1:24-27.

[7] Daniel F. Kiernan, Seenu M. Hariprasad. Normative Databases in SD-OCT: A Status Report. Normative Databases in SD-OCT: A Status Report: A comprehensive look at the evolution of OCT software design and database development. Fecha en que fue consultado: junio 20 de 2016.

[8] Theodossiadis GP, Boudouri A, Georgopoulos G. Central Visual Fields Changes after panretinal photocoagulation in proliferative diabetic retinopathy. Ophthalmologica 1990; 201(2):71-78

[9] Alexander J. Brucker. An Observational Study of the Development of Diabetic Macular, Edema Following Panretinal (Scatter) Photocoagulation (PRP) Given in 1 or 4 Sittings. Arch Ophthalmol. 2009; 127(2):132-140.

[10] H. Richard McDonald, Howard Shatz. Vissual loss followiing panretinal photocoagulation for proliferative diabetic retinopathy. Ophthalmology. 1985; 92(3):132-140.

[11] Einar Stefanson. The therapeutic effects of retinal laser treatment and vitrectomy. A theory base on oxygen and vascular physiology. Acta ophthalmol scand Suppl. 2001; 79:435-440.

[12] Katharina Kriechbaum, Matthias Bolz, Gabor G. Deak, Sonja Prager, Christopher Scholda, Ursula Schmidt-Erfurth. High resolution imaging of the human retina in vivo after scatter photocoagulation treatment using semiautomated laser system. Ophthalmology. 2010; 117(3); 545-551.

[13] Christoph Mitsch, Berthold Pemp, Katharina Kriechbaum, Matthias Bolz, Christoph Scholda, Ursula Schmidt-Erfurth. Retinal morphometry changes Measured with spectral Domain-optical coherence Tomography after pan-retinal Photocoagulation in patients With proliferative diabetic Retinopathy. Retina. 2016; 36(6); 1162-1169.

[14] Sung B. Lee, Joo Y. Kwag, Haeng J. Lee, Young J. Jo, Jung Y. Kim. The longitudinal changes of retinal nerve fiber layer thickness after panretinal Photocoagulation in diabetic retinopathy patients. Retina. 2013; 33:188-193. 
[15] Michele C. Lim, Suzana A. Tanimoto, Bruno A. Furlani, Brent Lum, Luciano M. Pinto, David Eliason. Effect of Diabetic Retinopathy and Panretinal Photocoagulation on Retinal Nerve Fiber Layer and Optic Nerve Appearance. Arch Ophthalmol. $2009 ; 127(7) ; 857-862$.

[16] Mahuil M. K. Muquit, Georger R. Marcelino, David B. Henson, Cecilia H. Fenerty, Paulo E. Stanga. Randomized clinical trial to evaluate the effects of pascal panretinal photocoagulation on macular nerve fiber layer, Manchester Pascal Study Report 3. Retina. 2011; 31(8):1669-1707.

[17] Faried M. Wagdy, Hoda M. El Sobky, Abd El-Rahman E. Sarhan, Mohammed A. Hafez. Evaluation of retinal nerve fiber layer thickness in diabetic retinopathy by optical coherence tomography after full scatter panretinal argon laser photocoagulation. J Egypt Ophthalmol Soc. 2013; 106:153-158.

[18] Hsu SY, Chung CP. Evaluation of retinal nerve fiber layer thickness in diabetic retinopathy after panretinal photocoagulation. Kaohsiung J Med Sci. 2002; 18(8):397-400.
[19] Michele C. Lim, Suzana A. Tanimoto, Bruno A. Purlani, Brent Lum, Luciano M Pinto, David Eliason, et al. Effect of Diabetic Retinopathy and Panretinal Photocoagulation on Retinal Nerve Fiber Layer and Optic Nerve Appearance. Arch ophthalmol. $2009 ; 127(7): 851-862$.

[20] Muqit MM, Sanghvi C, McLauchlan R, Delgado C, Young LB, Charles SJ, Marcellino GR, Stanga PE. Study of clinical applications and safety for Pascal ${ }^{\circledR}$ laser photocoagulation in retinal vascular disorders. Acta ophthalmol. 2012; 90(2):155-161.

[21] Yi-Ryeung Park, Donghyun Jee. Changes in Peripapillary Retinal Nerve Fiber Layer Thickness after Pattern Scanning Laser Photocoagulation in Patients with Diabetic Retinopathy. Korean J Ophthalmol. 2014; 28(3):220-225.

[22] Giacomo Savini, Piero Barboni, Michele Carbonelli, Alessandra Shreglia, Giulia Deluigi. Comparison of optic nerve head parameter measurement obtained by time-domain and Spectral-domain optical coherence tomography. Glaucoma, 2013; 22(5):385-389. 